\title{
Modeling Neural Injury in Organotypic Cultures by Application of Inertia-Driven Shear Strain
}

\author{
MICHAEL BOTTLANG, ${ }^{1}$ MARK B. SOMMERS, ${ }^{1}$ THERESA A. LUSARDI, ${ }^{2}$ \\ JENNIFER J. MIESCH, ${ }^{2}$ ROGER P. SIMON, ${ }^{2}$ and ZHI-GANG XIONG ${ }^{2}$
}

\begin{abstract}
In vitro models of traumatic brain injury (TBI) are indispensable to explore the effects of mechanotrauma on neurological injury cascades and injury thresholds. This study characterizes a novel in vitro model of neural shear injury, which for the first time subjects organotypic cultures to inertiadriven shear strain. In this model, organotypic cultures preserved a high level of biological heterogeneity and spatial cytoarchitecture, while inertia-driven shear strain represented a tissue-level insult typical for closed head TBI in vivo. For neural injury simulation, organotypic hippocampal cultures derived from rats were inserted in an inertial loading module, which was subjected to impacts at five graded impact velocities ranging from 2 to $10 \mathrm{~m} / \mathrm{sec}$. The mechanical insult was quantified by measuring the transient shear deformation of the culture surface during impact with a high-speed camera. The resultant cell death was quantified with propidium iodide (PI) staining 24 hours following shear injury. Increasing impact velocities of $2,4.6,6.6,8.1$, and $10.4 \mathrm{~m} / \mathrm{sec}$ caused graded peak shear elongation of $2.0 \pm 0.9 \%, 5.4 \pm 3.8 \%, 15.1 \pm 14.6 \%, 25.4 \pm 14.7 \%$, and $56.3 \pm$ $51.3 \%$, respectively. Cell death in response to impact velocities of $6.6 \mathrm{~m} / \mathrm{sec}$ or less was not significantly higher than baseline cell death in sham cultures $(4.4 \pm 1.5 \%)$. Higher impact velocities of 8.1 and $10.4 \mathrm{~m} / \mathrm{sec}$ resulted in a significant increase in cell death to $19.9 \pm 12.9 \%$ and $36.7 \pm 14.2 \%$, respectively $(p<0.001)$. The neural shear injury model delivered a gradable, defined mechanotrauma and thereby provides a novel tool for investigation of biological injury cascades in organotypic cultures.
\end{abstract}

Key words: brain injury; hippocampus; organotypic; shear strain; TBI

\section{INTRODUCTION}

$\mathbf{L}$ ABORATORY MODELS of traumatic brain injury (TBI) $\Delta$ are indispensable tools for the investigation of brain injury mechanisms and exploration of neuroprotective treatment strategies. A wide variety of in vivo (DenneyBrown and Russel, 1943; Ommaya and Gennarelli, 1974;
Gennarelli et al., 1982; Lighthall et al., 1989; Meaney et al., 1994; Gutierrez et al., 2001) and in vitro TBI models (Ellis et al., 1995; Cargill and Thibault, 1996; LaPlaca et al., 1997; Morrison et al., 1998a, 1998b; Geddes and Cargill, 2001) have been introduced to correlate mechanical insults with the consequential biological injury cascades in brain. In common, these TBI models require

\footnotetext{
${ }^{1}$ Biomechanics Laboratory and ${ }^{2}$ Robert S. Dow Neurobiology Laboratories, Legacy Research, Portland, Oregon.
} 
generation of a clinically meaningful mechanical insult that can be characterized and reproduced.

In vivo TBI models provide superior biofidelity. However, the complexity of TBI simulation in animals necessarily limits the ability to characterize and spatially delineate the physical insult and the consequential injury cascade. In vitro TBI models based on two-dimensional neuronal cell cultures allow assessment of specific injury response parameters on the cellular and molecular level in a controlled environment (Ellis et al., 1995; Cargill and Thibault, 1996; LaPlaca et al., 1997, 2005; Muhkin et al., 1997; Smith et al., 1999; Geddes and Cargill, 2001; Lusardi et al., 2004), but lack the biofidelity of in vivo models. Most recently, organotypic brain slice cultures have been adopted for in vitro TBI modeling (Gaehwiler et al., 1997; Muhkin et al., 1997; Morrison et al., 1998a,b; Adamchik et al., 2000; DeRidder et al., 2001; Cater et al., 2004). Organotypic brain cultures preserve the heterogeneous cell population and cytoarchitecture of brain in a controlled culture environment over a prolonged period of time (Stoppini et al., 1991, 1997). As such, they are uniquely suited for TBI simulation in vitro by combining a high degree of biofidelity with the ability to deliver a controlled mechanical insult.

The first organotypic TBI experiments induced mechano-trauma by rolling a steel pin over cultures and by dropping a weight onto cultures (Adamchik et al., 2000). Subsequent organotypic TBI models utilized controlled distention of elastic culture substrates to induce stretch injury (Morrison et al., 1998a, 2000; DeRidder et al., 2001). Initially, these systems utilized vacuum pulses for sinusoidal downward deflection of culture substrates, generating membrane stretch up to $65 \%$ as well as superimposed out of plane acceleration (Morrison et al., 1998a, 2000). Membrane stretch models have successively been refined to provide for pure in-plane stretch in the absence of out-of-plane acceleration (Cater et al., 2004). This was accomplished by pulling the perimeter of a distensible substrate over a fixed cylinder, effectively reducing the mechanical insult to in-plane stretch of the organotypic culture. While these TBI models can deliver controlled stretch injury, no organotypic TBI model to date has been designed to induce controlled mechanotrauma in the form of shear injury.

Inertia-driven shear strain is believed to be a governing mechanism in closed-head TBI, caused by rapid acceleration or deceleration of a head during impact (Gennarelli et al., 1982; Meaney et al., 1995; King et al., 2003). For TBI modeling in vitro, the biological injury cascade should plausibly be initiated by a mechanical stimulus most relevant to TBI causation in vivo. Therefore, we developed an organotypic neural shear injury (NSI) model which employs inertia-driven shear strain to generate scalable mechanotrauma. Specifically, we hypothesized that scalable shear deformation and consequential cell injury in organotypic brain slices can be generated by impact-induced inertial loading. Subsequently, we characterized the NSI model by correlating shear deformation of the organotypic cultures to cellular death for a range of injury severities.

\section{METHODS}

\section{Organotypic Culture}

Organotypic hippocampal slices from postnatal Wistar rats were cultured using the interface method (Stoppini et al., 1991). The use of rat brain for slice culture was approved by the Institutional Animal Care and Use Committee of Legacy Clinical Research and Technology Center. Whole brains from 6-8-day-old rats were removed under anesthesia with halothane. The brains were placed in sterile, ice-cold dissecting medium (Hanks BSS, $5 \mathrm{mg} / \mathrm{ml}$ glucose, $20 \mathrm{mM}$ HEPES) for $5 \mathrm{~min}$ prior to dissection. Hippocampi and the adjacent cortical regions were dissected out, then sectioned in $400-\mu$ m-thick slices (McIlwain ${ }^{\mathrm{TM}}$, Stoelting Co., Wood Dale, IL) and floated into dissecting media. Slices were trimmed to the hippocampus and adjacent entorhinal cortex, and then transferred to Millicell culture plate inserts with microporous membranes (PICM0RG50; Millipore, Bedford, MA; Fig. 1a). Inserts were preincubated in culture medium containing 50\% MEM, 25\% horse serum, 25\% HBSS, $20 \mathrm{mM}$ HEPES, $1 \mathrm{mM}$ l-glutamine (Glutamax; Invitrogen, Carlsbad, CA), and $25 \mathrm{mM}$ glucose. The slices were maintained in an incubator at $36^{\circ} \mathrm{C}$ with a $5 \%$ $\mathrm{CO}_{2}$ enriched, humidified atmosphere. Medium was changed the day after plating and every $3-4$ days after.

\section{Neural Shear Injury Model}

An NSI system was developed that subjected organotypic cultures to transient shear deformation by linear impact-induced inertial loading. After stabilizing in vitro for 12 days, hippocampal slices on Millicell inserts were temporarily transferred to an inertial loading module (Fig. 1b) for induction of neural shear injury. This custom module was made of polystyrene, commonly used in cell culture environments (Curtis et al., 1983; Steele et al., 1993). The module holds up to six Millicell inserts in precision-machined pockets in a standard six-well arrangement. A translucent Plexiglas lid on the module protected the cultures against contamination and airflow during experiments.

This inertial loading module was inserted into the linear slide of the NSI system to rest against the pis- 


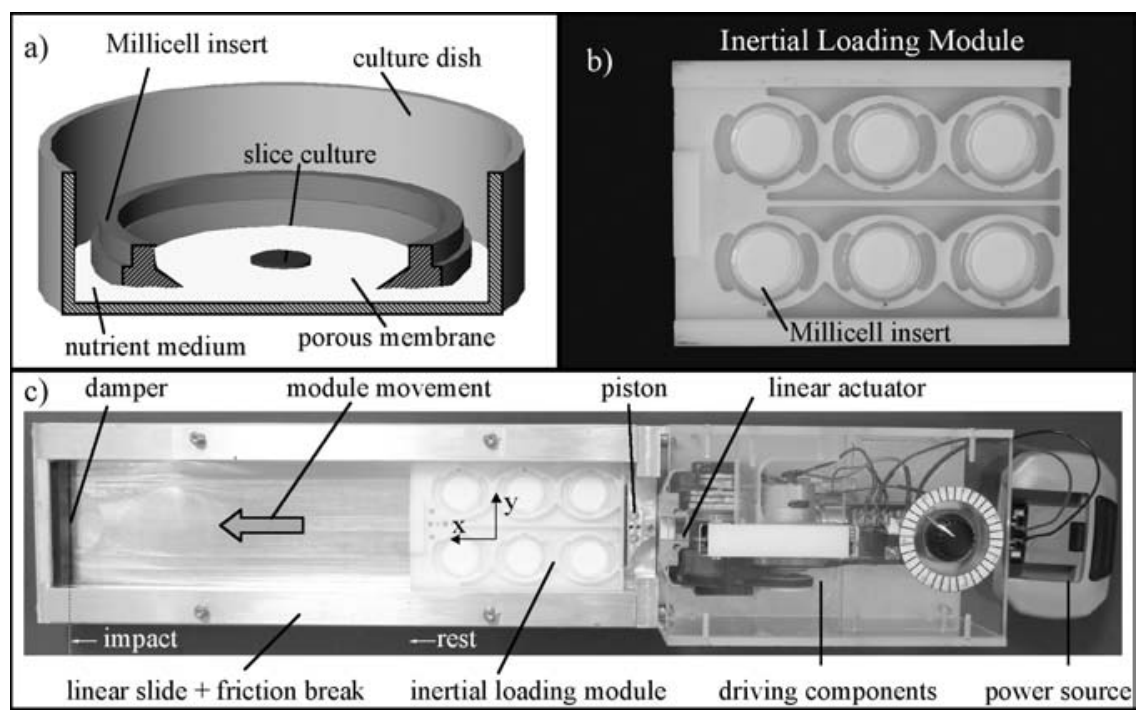

FIG. 1. (a) Hippocampal slices were maintained as organotypic interface cultures on Millicell inserts in a culture dish. (b) After 12 days in vitro, Millicell inserts with hippocampal cultures were temporarily transferred into the inertial loading module containing six Millicell inserts. (c) For shear injury simulation, the module was inserted into the linear slide of the NSI system.

ton of a linear actuator (Fig. 1c). The actuator accelerated the module over a 90 -mm stroke length. The electronically controlled actuator was driven by a flywheel mechanism engaged by a solenoid-powered clutch. Impact injury was induced after $260-\mathrm{mm}$ travel, where the inertial loading module impacted a 6-mm-thick elastomer damper of 80 Durometer shore hardness. The rapid deceleration during impact induced an inertia-driven shear deformation of hippocampal cultures. By modulating the energy release of the actuator, five graded impact velocities $v_{I}$ were attained. In pilot studies, the highest impact velocity was experimentally determined to be just below an impact velocity that yielded complete shear-disruption of organotypic cultures. This impact velocity was termed $\mathrm{v}_{100 \%}$. The remaining four impact velocities were scaled to attain nominal impact velocities in $20 \%$ increments of $\mathrm{v}_{100 \%}$ (i.e., $\mathrm{v}_{20 \%}, \mathrm{v}_{40 \%}, \mathrm{v}_{60 \%}, \mathrm{v}_{80 \%}$ ). After impact, an adjustable friction brake integrated in the linear slide controlled the rebound of the inertial loading module and ensured that the module would come to halt before contacting the retracted actuator.

The battery powered NSI system was designed to fit into a laminar flow culture hood. The NSI system was furthermore designed to ensure easy handling, allowing completion of experiments with up to six culture wells in less than one minute. For this period of time only, culture medium was removed from underneath the culture membranes to prevent fluid shear stress during NSI simulation.

\section{Shear Insult Characterization}

The thickness of hippocampal cultures and their transient deformation during impact was measured to quantify the inertia-driven shear insult induced in hippocampal cultures. Culture thickness required assessment since hippocampal slices do not preserve their initial thickness but undergo progressive thinning in culture. To measure the culture thickness $h$, the culture was transferred on a microscope with a $\mathrm{z}$-axis micrometer stage connected to a digital readout. The microscope was first focused on cells on the Millicell membrane, and subsequently on cells on the surface layer of the hippocampal culture. The distance between the focus planes represented the culture thickness $h$, specific for each hippocampal culture tested.

Transient deformation of the culture surface during impact was captured using a high-speed camera system (Fastcam Ultima APX RS; Photron, Itronx, CA; Fig. 2a). The camera and a glass-fiber guided cold illumination light (Novoflex; WPI, Sarasota, FL) were positioned statically over the linear slide to capture the hippocampal culture during impact. Images with $512 \times 128$ pixels per frame were obtained at 36,000 frames/sec. A zoom lens (AF Micro Nikkor; Nikon, Melville, NY) was used to gain a spatial resolution of $19 \mu \mathrm{m} /$ pixel. Since both the Millicell membrane and the hippocampal culture are translucent, markers were used to track the transient shear displacement of the hippocampal surface relative to the Millicell membrane (Fig. 2b). An $18-\mu \mathrm{m}$ thin-film marker approximately $1 \times 2 \mathrm{~mm}$ in size was centered on 


\section{MODELING NEURAL INJURY IN ORGANOTYPIC CULTURES}

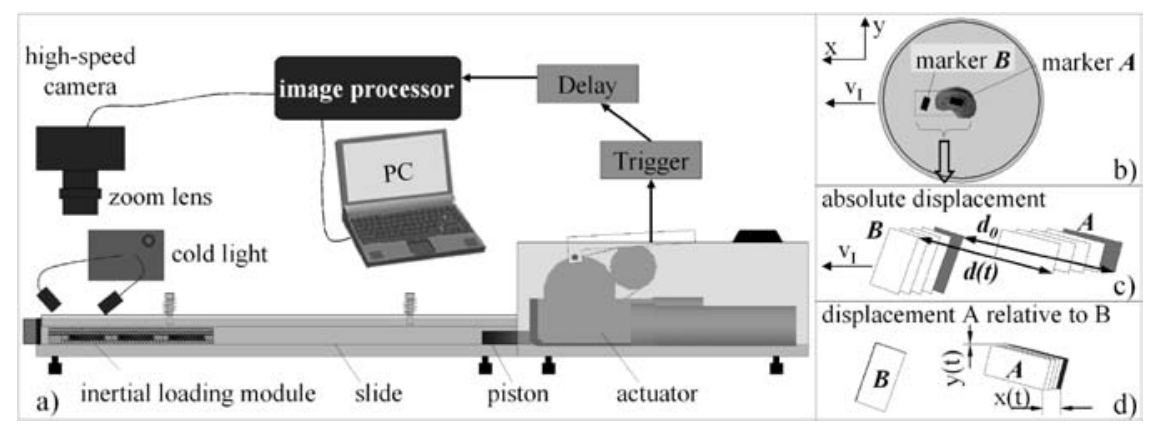

FIG. 2. (a) Assessment of transient shear deformation with a high-speed camera system. (b) markers were placed on the culture (A) and on the membrane (B) to acquire shear displacement of the culture surface relative to the Millicell membrane. (c) Initial distance $d_{0}$ between markers $\mathrm{A}$ and $\mathrm{B}$, and apparent distance $d(t)$ during impact-induced shear deformation. (d) Displacement of marker A relative to marker B parallel $(x)$ and perpendicular $(y)$ to the loading direction.

the hippocampus surface using microsurgery forceps and magnifying optics. The marker backing contained a water-based gelatin adhesive for bonding with the culture surface. The marker was considered neutrally buoyant, since its density of $0.9 \mathrm{~g} / \mathrm{cm}^{3}$ was just below that of brain matter (Zhang et al., 2002). As reference for shear assessment, a second marker was affixed on the Millicell membrane adjacent to the hippocampal culture. Shear deformation of the hippocampal culture was measured by tracking changes in the in-plane distance $\boldsymbol{d}_{x, y}$ between the markers on high-speed videos, using ImageJ software (NIH). Each high-speed image was discretized at a fixed gray-scale threshold for semi-automated edge detection and corner interpolation. First, the impact velocity $v_{I}$ was calculated from the displacement history of the membrane-fixed reference marker immediately before impact. Next, the baseline distance $\boldsymbol{d}_{0}$ for the unloaded specimens was measured on a static image, obtained before shear injury simulation (Fig. 2c). Subsequently, time-displacement histories of relative shear displacement components $x(t)=d_{x}(t)-d_{x}(t=0)$ and $y(t)=d_{y}(t)-d_{y}(t=0)$ between the hippocampal surface and its membrane substrate were extracted (Fig. 2d). Finally, residual shear displacement $x_{\text {res }}$ and $y_{\text {res }}$ were extracted from a static image, obtained after the inertial loading module came to halt.

Shear deformation was characterized in terms of longitudinal elongation $E_{L}$ derived from a generalized formulation of Green's strain tensor (Fung, 1994). The finite elongation $E_{L}$ was therefore calculated by

$$
\begin{aligned}
& E_{L}=\left(\sqrt{2 E_{33}+1}-1\right) \times 100 \% \text {, whereby } \\
& \qquad E_{33}=\frac{1}{2} \times \frac{x^{2}+y^{2}}{h^{2}}
\end{aligned}
$$

with $x$ being the maximal finite displacement component of the culture surface relative to the membrane substrate, $y$ being the absolute displacement perpendicular to the loading direction $\mathrm{x}$, and $h$ being the culture thickness. Components $\mathrm{E}_{33}$ and $\mathrm{E}_{13}$ of the Green's strain tensor were derived in addition for result comparison with prior research:

$$
\begin{aligned}
E_{33}=\frac{1}{2}\left(\left(\frac{\partial x_{1}}{\partial a_{2}}\right)^{2}+\left(\frac{\partial x_{2}}{\partial a_{2}}\right)^{2}\right) & =\frac{1}{2} \times \frac{x^{2}+y^{2}}{h^{2}} \\
E_{13} & =\frac{1}{2}\left(\frac{\partial x_{1}}{\partial a_{1}} \frac{\partial x_{1}}{\partial a_{2}}\right)=\frac{1}{2} \times \frac{x}{h} .
\end{aligned}
$$

\section{Cellular Injury Characterization}

Cell viability was assessed for all hippocampal regions combined and region-specific (i.e., CA1, CA3, and dentate gyrus) on a total of 119 cultures in response to the five graded injury severities. Seventeen cultures were used as control and remained in the incubator for the entire period of the experiment. Nineteen sham cultures were removed from the incubator on the day of shear injury simulation and temporarily transferred to the inertial loading module without being subjected to inertial loading. Eighty-three cultures were subjected to the five graded impact velocities: $\mathrm{v}_{20 \%}(n=15), \mathrm{v}_{40 \%}(n=16)$, $\mathrm{v}_{60 \%}(n=15), \mathrm{v}_{80 \%}(n=19)$, and $v_{100 \%}(n=18)$.

The amount of cell death was measured by propidium iodide uptake (PI; Molecular Probes) $24 \mathrm{~h}$ following injury. Slices were incubated in PI $(2 \mu \mathrm{M})$ containing medium for $3 \mathrm{~h}$, then imaged using an upright microscope (Leica DM R, Wetzlar, Germany) with a Retiga 1300 camera (QImaging) at $100 \mathrm{msec}$ exposure time. Each slice was normalized to a maximum injury condition triggered by exposure to $1 \mathrm{mM}$ NMDA $+10 \mu \mathrm{M}$ glycine in culture medium for $24 \mathrm{~h}$ to induce cell death of all neurons. Subsequently, each slice was imaged a second time under the same exposure to allow calcula- 


\section{BOTTLANG ET AL.}

tion of a percentage cell death. Using Image $(\mathrm{NIH})$, the average intensity of the CA1, CA3, and dentate gyrus region on each slice was measured at $24 \mathrm{~h}$ after impact and after maximum injury with NMDA. Reported values are the 24-h time point divided by the maximum intensity for the same slice.

\section{Statistical Analysis}

Statistical analysis of neural cell death in correlation to peak elongation was performed using analysis of variance (ANOVA) at a confidence level of $\alpha=0.05$ with a Scheffe's posthoc test to account for unequal variances and unequal number of specimens. All results were calculated representing mean values \pm one standard deviation.

\section{RESULTS}

The thickness $h$ of organotypic cultures decreased after 12 days in vitro by $44 \%$ to $225 \pm 35 \mu \mathrm{m}$. High-speed images successfully captured the transient displacement history of the culture surface relative to the Millicell membrane during impact. Reproducibility of semi-automated image analysis was demonstrated by three repeat evaluations at the highest impact velocity of one highspeed recording, yielding a velocity $\mathrm{v}_{100 \%}$ of $10.5 \pm 0.02$ $\mathrm{m} / \mathrm{sec}$ and a maximal displacement $x$ of $195 \pm 26 \mu \mathrm{m}$.

On average, impact velocities derived from the membrane-fixed marker displacement were $\mathrm{v}_{20 \%}=2.0 \pm$ $0.8 \mathrm{~m} / \mathrm{sec}, \mathrm{v}_{40 \%}=4.6 \pm 0.4 \mathrm{~m} / \mathrm{sec}, \mathrm{v}_{60 \%}=6.6 \pm 0.4$ $\mathrm{m} / \mathrm{sec}, \mathrm{v}_{80 \%}=8.1 \pm 0.2 \mathrm{~m} / \mathrm{sec}$, and $\mathrm{v}_{100 \%}=10.4 \pm$ $0.3 \mathrm{~m} / \mathrm{sec}$. The impact-induced peak shear displacement was $\mathrm{x}_{20 \%}=37 \pm 6.1 \mu \mathrm{m}, \mathrm{x}_{40 \%}=71.2 \pm 17.2 \mu \mathrm{m}$, $\mathrm{x}_{60 \%}=99.6 \pm 71.7 \mu \mathrm{m}, \mathrm{x}_{80 \%}=167.7 \pm 58.2 \mu \mathrm{m}$, and

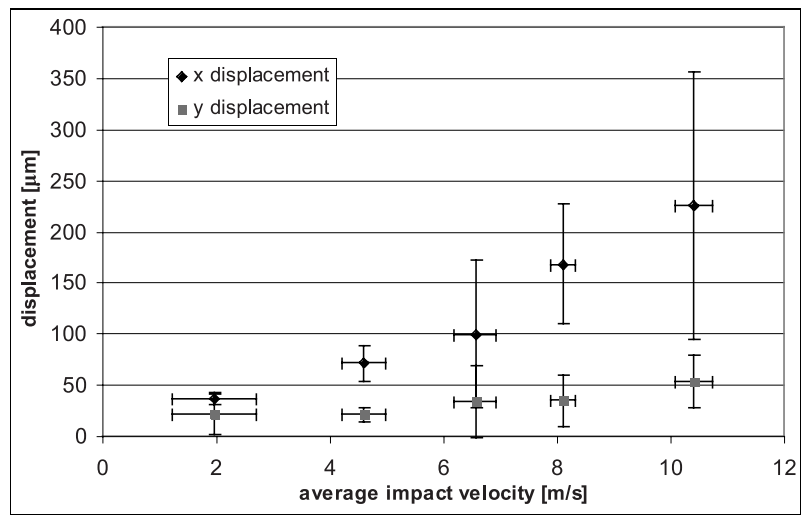

FIG. 3. Average and standard deviation of peak displacement in $x$ and $y$ direction in response to the five graded impact velocities.

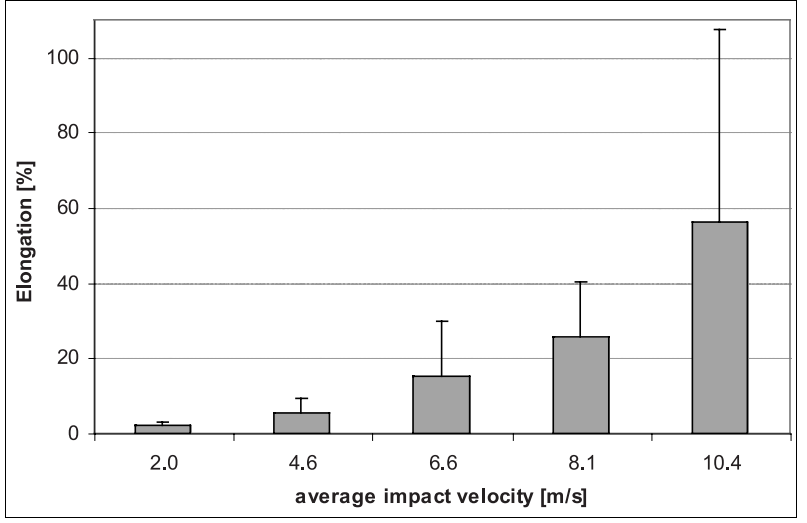

FIG. 4. Peak elongation increased for increasing impact velocities (mean $+\mathrm{SD})$.

$\mathrm{x}_{100 \%}=226 \pm 131 \mu \mathrm{m}$ (Fig. 3). Absolute displacement in the $y$-direction perpendicular to the loading direction $x$ was on average $21.5 \pm 19.3 \mu \mathrm{m}, 20.7 \pm 6.8 \mu \mathrm{m}$, $33.8 \pm 35.0 \mu \mathrm{m}, 34.2 \pm 25.4 \mu \mathrm{m}$, and $53.6 \pm 26.0 \mu \mathrm{m}$ for impacts at $\mathrm{v}_{20 \%}, \mathrm{v}_{40 \%}, \mathrm{v}_{60 \%}, \mathrm{v}_{80 \%}$, and $\mathrm{v}_{100 \%}$, respectively. The duration from onset of shear displacement to its peak was on average $0.43,0.44,0.39,0.46$, and $0.42 \mathrm{msec}$ for impacts at $\mathrm{v}_{20 \%}, \mathrm{v}_{40 \%}, \mathrm{v}_{60 \%}, \mathrm{v}_{80 \%}$, and $v_{100 \%}$, respectively. Each of these deformation histories was captured on image sequences that contained over 30 frames. After impact, no culture demonstrated non-recoverable, residual shear deformation indicative of structural failure.

Peak elongation $E_{L}$ increased with impact velocity (Fig. 4). Impact velocities of $\mathrm{v}_{20 \%}=2 \mathrm{~m} / \mathrm{sec}, \mathrm{v}_{40 \%}=4.6$ $\mathrm{m} / \mathrm{sec}, \mathrm{v}_{60 \%}=6.6 \mathrm{~m} / \mathrm{sec}, \mathrm{v}_{80 \%}=8.1 \mathrm{~m} / \mathrm{sec}$, and $\mathrm{v}_{100 \%}=$ $10.4 \mathrm{~m} / \mathrm{sec}$ yielded peak elongations $E_{L, \text { peak }}$ of $2.0 \pm$ $0.9 \%, 5.4 \pm 3.8 \%, 15.1 \pm 14.6 \%, 25.4 \pm 14.7 \%$, and $56.3 \pm 51.3$, respectively. For correlation of outcome parameters, a summary of results for $v_{I}, \Delta t, h, x_{\text {peak }}, y_{\text {peak }}$, $E_{L, \text { peak }}, E_{13 \text {,peak }}$, and $E_{33 \text {,peak }}$ is provided in Table 1.

Cell death assessment with PI in all regions yielded no significant difference between control cultures (3.16 \pm $1.6 \%)$ and sham cultures $(4.43 \pm 1.54 \%)$ (Fig. 5a). Impacts of up to $\mathrm{v}_{40 \%}=4.6 \mathrm{~m} / \mathrm{sec}$ and $\mathrm{E}_{\mathrm{L} \text {,peak }}=5 \%$ did not cause additional cell death. Impacts of $\mathrm{v}_{60 \%}=6.6 \mathrm{~m} / \mathrm{sec}$ and $\mathrm{E}_{\mathrm{L} \text {,peak }}=15 \%$ caused $10.1 \pm 10.6 \%$ cell death, though this increase in cell death over sham cultures was not significant. Impacts at $\mathrm{v}_{80 \%}=8.1 \mathrm{~m} / \mathrm{sec}\left(\mathrm{E}_{\mathrm{L}, \text { peak }}=\right.$ $25 \%)$ and $\mathrm{v}_{100 \%}=10.4 \mathrm{~m} / \mathrm{sec}\left(\mathrm{E}_{\mathrm{L}, \text { peak }}=56 \%\right)$ significantly elevated cell death to $19.9 \pm 12.9 \%$ and $36.7 \pm$ $14.2 \%$ ( $p<0.001$ ), respectively (Fig. 5a,b). Region-specific evaluation did not produce different statistical results. However, at impact velocities of $6.6 \mathrm{~m} / \mathrm{sec}$ or higher, the CA1 region consistently exhibited the most cell death. 
Table 1. Summary of Outcome Parameters Characterizing the Inertia-Driven Shear Insult Delivered to Hippocampal Cultures $\left(n=6\right.$ Per $\left.v_{I}\right)$

\begin{tabular}{|c|c|c|c|c|c|c|c|c|c|c|c|}
\hline & & \multicolumn{10}{|c|}{ Nominal impact velocity } \\
\hline \multicolumn{2}{|c|}{ Outcome } & \multicolumn{2}{|c|}{$V_{20 \%}$} & \multicolumn{2}{|c|}{$V_{40 \%}$} & \multicolumn{2}{|c|}{$V_{60 \%}$} & \multicolumn{2}{|c|}{$V_{80 \%}$} & \multicolumn{2}{|c|}{$V_{100 \%}$} \\
\hline Parameter & Unit & Average & Stdev & Average & Stdev & Average & Stdev & Average & Stdev & Average & Stdev \\
\hline$v_{I}$ & {$[\mathrm{~m} / \mathrm{s}]$} & 2.0 & 0.8 & 4.6 & 0.4 & 6.6 & 0.4 & 8.1 & 0.2 & 10.4 & 0.3 \\
\hline$\Delta t$ & {$[\mathrm{~ms}]$} & 0.4 & 0.3 & 0.4 & 0.1 & 0.4 & 0.1 & 0.5 & 0.1 & 0.4 & 0.0 \\
\hline$h$ & {$[\mu \mathrm{m}]$} & 230.8 & 39.7 & 242.0 & 37.4 & 209.3 & 32.4 & 235.7 & 23.3 & 213.2 & 39.5 \\
\hline$x_{\text {peak }}$ & {$[\mu \mathrm{m}]$} & 37.2 & 6.1 & 71.2 & 17.2 & 99.6 & 71.7 & 167.7 & 58.2 & 225.5 & 131.0 \\
\hline abs $y_{\text {peak }}$ & {$[\mu \mathrm{m}]$} & 21.5 & 19.3 & 20.7 & 6.8 & 33.8 & 35.0 & 34.2 & 25.4 & 53.6 & 26.0 \\
\hline$E_{13, \text { peak }}$ & {$[\%]$} & 8.3 & 2.2 & 15.3 & 5.6 & 23.3 & 15.5 & 35.7 & 12.3 & 54.5 & 34.8 \\
\hline$E_{33 \text {,peak }}$ & {$[\%]$} & 2.0 & 1.0 & 5.6 & 4.1 & 17.1 & 17.5 & 29.5 & 18.7 & 83.1 & 86.4 \\
\hline$E_{L, p e a k}$ & {$[\%]$} & 2.0 & 0.9 & 5.4 & 3.8 & 15.1 & 14.6 & 25.4 & 14.7 & 56.3 & 51.3 \\
\hline
\end{tabular}

\section{DISCUSSION}

The presented organotypic NSI model combines a high level of biological complexity with an inertia-driven shear insult that resembles the tissue level mechanotrauma believed to govern closed head TBI in vivo (Gennarelli et al., 1982; King et al., 1995; Meaney et al., 1995). It therefore combines principal biological and mechanical aspects for simulation of neural shear injury in a controlled environment.

For in vitro TBI simulation, organotypic cultures offer distinct advantages over dissociated cell cultures that are typically harvested by mechanical or enzymatic dissociation, which severs endogenous connections (Morrison et al., 1998b). Organotypic cultures maintain many aspects of the intrinsic connectivity, neuronal and glial organization, and physiological characteristics of intact brain tissue (Stoppini et al., 1991; Hailer et al., 1996; Abdel-Hamid and Tymianski, 1997; Krimer and GoldmanRakic, 1997; Stoppini et al., 1997). Although the density of synaptic structures in hippocampal slices decreases after slicing (Hailer et al., 1996), subsequent development of synaptic structures parallels synaptogenesis in situ (Finsen et al., 1992; Buchs et al., 1993; Mielke et al.,

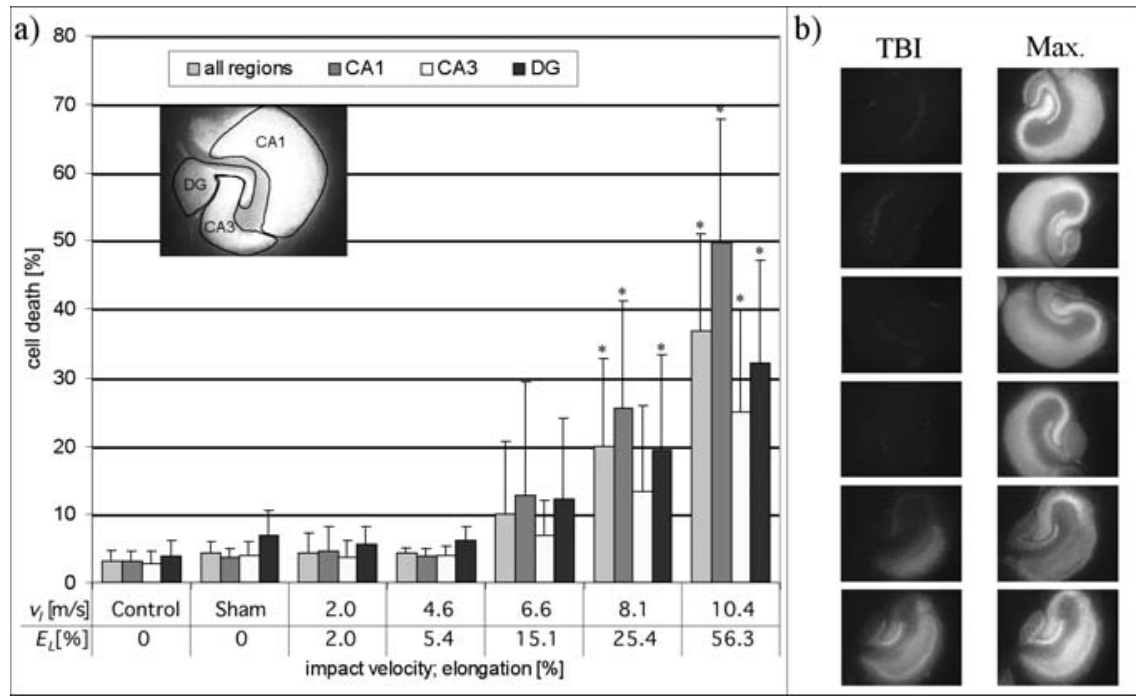

FIG. 5. (a) Cell death in all regions combined and region-specific (CA1, CA3 and dentate gyrus), caused by the mechanical insult and measured by PI uptake (mean $+\mathrm{SD}$ ). No difference in cell death was observed between control and sham cultures. Compared to sham, significant increases in cell death were detected at and above $\mathrm{v}_{\mathrm{I}}=8.1 \mathrm{~m} / \mathrm{sec}$ and $\mathrm{E}_{\mathrm{L}, \mathrm{peak}}=25 \%$. $* p<0.05$. (b) Representative images of PI-uptake in hippocampal cultures subjected to increasing impact velocities (left column) and maximum neural cell death after NMDA treatment (right column). 


\section{BOTTLANG ET AL.}

2005). Direct comparison of age-matched organotypic cultures with acute slices demonstrated parallel synaptic development, confirming that hippocampal synaptic development does not rely on environmental cues absent in vitro (De Simoni et al., 2003). Furthermore, comparison between organotypic hippocampal cultures and in vivo models show good correlation across various stimuli, including models of hypoxia/ischemia (Moroni et al., 2002) and agonist/drug exposure (Elliott-Hunt et al., 2004). Moreover, the expression and distribution of glutamate receptors are very similar to those in adult tissue and several important proteins, such as synaptophysin, microtubule-associated proteins and neural cell adhesion molecules are expressed at steady levels (Bahr et al., 1995). Viability of organotypic cultures of up to two months can be obtained by the interface culture technique, in which acute brain slices are placed on a porous membrane at the air-medium interface (Stoppini et al., 1991). This well-established interface technique has been chosen as the basis for the present organotypic NSI model, providing robust culture viability with routine culture techniques in commercially available Millicell wells.

Cell death assessment with PI yielded no significant difference between control cultures $(3.16 \pm 1.6 \%)$ and sham cultures $(4.43 \pm 1.54 \%)$, demonstrating that the experimental procedure of transitory nutrient deprivation and culture transfer into the NSI system did not affect culture viability. The presented organotypic NSI model delivered a controlled, reproducible impact velocity resulting in inertia-driven shear deformation. Inertial loading in a controlled manner was induced by impact velocities with less than $3 \%$ standard deviation at the highest impact velocity setting. The peak deceleration during impact was over 10 times higher than the acceleration by the electromechanical actuator, measured in a preliminary test. This suggests that neural shear injury was primarily confined to the impact scenario, and not to the initial acceleration prior to impact. Nevertheless, the acceleration provided by the electromechanical actuator was essential to reach desired impact velocities in a distance that allows for shear injury modeling within the geometric confines of a conventional laminar flow hood.

Mechanotrauma in organotypic cultures was for the first time induced by inertial loading. Given a nominally uniform density of brain tissue, inertial load vectors will act equally on each volume element within the organotypic culture according to Newton's second law. Therefore, the entire culture volume was subjected to shear deformation. Shear is believed to be the central mechanism underlying neuronal death and diffuse axonal injury in closed head TBI (Gennarelli et al., 1982; Meaney et al., 1994; King et al., 1995). As an outcome parameter for finite shear displacement, elongation was selected over unit-less shear strain or alternative strain tensor components since it directly represents a physical measure of finite deformation in terms of percentage strain.

Previous organotypic TBI models typically employed stretch by distention of a flexible culture membrane as the principal mechanism of mechanotrauma (Morrison et al., 1998a; DeRidder et al., 2001; Cater et al., 2004). Morrison et al. were the first to thoroughly characterize the bi-axial strain fields on the distensible membrane of their stretch-induced TBI model (Morrison et al., 1998a). For their vacuum-driven TBI system, they measured static and dynamic pressure versus deflection of the culture membrane and provided an experimental validation of a closed-form analytical expression relating membrane deflection to radial strain. Their system induced up to $65 \%$ stretch, in addition to superimposed inertial loading by membrane displacement perpendicular to the direction of the intended mechanical strain stimulus. In order to reduce superimposed inertial loading and bi-axial strain heterogeneity, DeRidder et al. supported the distensible membrane with a slotted steel plate (DeRidder et al., 2001). Therefore, membrane pressurization stretched only the membrane segment overlying the slot, yielding primarily uni-directional membrane stretch of up to $70 \%$ under highly reduced out-of-plane deflection. Cater et al. introduced an organotypic TBI model capable of substrate stretching in the absence of deflection by pulling the perimeter of a silicone membrane over a fixed rim (Cater et al., 2004). These stretch-based organotypic TBI models employ a defined substrate strain under the assumption of dynamic strain propagation into the organotypic culture. Most recently, La Placa et al. introduced a 3-D cell shearing device, in which astrocytes suspended in Matrigel were subjected to shear strain of up to 0.5 (LaPlaca et al., 2005). Although their model did not incorporate the heterogeneous cell distribution preserved in organotypic cultures, they characterized clinically relevant shear injury of 3-D cultures in response to a welldefined mechanotrauma.

Previous TBI models, based on distensible membrane stretch, found that injury thresholds depended on the magnitude and rate of the applied insult, as well as on the cell type. Neuronal monolayers exhibited strain and strain rate dependent alterations in intracellular calcium in response to 10-30\% strain (Cargill and Thibault, 1996; Geddes and Cargill, 2001; Lusardi et al., 2004). Astrocytes proved highly resilient to stretch, requiring a stretch injury of $72 \%$ strain to yield 35\% cell death (Ellis et al., 1995). Axons were found to have a remarkably high tolerance to stretch as compared to neurons, withstanding strains below 65\% in the absence of primary axotomy (Smith et al., 1999). Organotypic cultures subjected to substrate stretch of $40 \%$ exhibited $30 \%$ cell death, as 
compared to $15 \%$ cell death in control cultures (Morrison et al., 2000). Stretch of $70 \%$ induced both apoptotic and necrotic cell death within $24 \mathrm{~h}$ (DeRidder et al., 2001). Similar to previous studies, we found significantly elevated cell death of $20 \%$ and $37 \%$ in response to shear elongations of $25 \%$ and $56 \%$, respectively. Therefore, the NSI model of inertia-driven shear strain constitutes a novel approach for injury simulation in brain tissue with a defined, clinically pertinent mechanotrauma.

This NSI model has several limitations. It does not attempt to replicate the complex, heterogeneous distortion patterns expected in vivo, but instead simulates a defined, uniaxial shear deformation, representing tissue level shear distribution as a unique and relevant aspect of mechanotrauma. The reported shear elongation did not account for strain gradients over the thickness of the brain slice, but represents only the average shear strain observed between the top and bottom layer of the culture. For shear deformation assessment, uniform displacement of the surface layer under inertial loading was assumed, based on a nominally uniform density of the hippocampal slice.

Finite shear deformation was characterized in terms of longitudinal elongation $E_{l}$ derived from Green's strain tensor. Given the incompressibility of brain tissue, outof-plane displacement can reasonably assumed to be negligible. Attempts were made to visually orient hippocampal cultures with the CA1 region parallel to the loading direction, but accurate alignment in a timely manner proved difficult and was therefore not formally controlled. Future studies should employ a formal mechanism to facilitate culture alignment, which would enable investigation of the effects of loading direction on shear elongation and cell injury. Unlike impact velocity, shear elongation exhibited appreciable standard deviations, which may be caused by variances in thickness, and constitutive properties of organotypic cultures. Finally, the dose-response characterization of the model focused only on the correlation between cell death and peak shear elongation, while the duration from onset to peak of elongations was kept nearly constant $(0.4 \mathrm{~ms}-0.5 \mathrm{~ms})$. This duration was of the same order in magnitude than impact durations of $1.2 \pm 0.1 \mathrm{~ms}$ reported for a pendulum impact model of neuronal monolayer cultures (Lucas and Wolf, 1991). Furthermore, the duration was nominally one order of magnitude shorter than durations typical for stretch-induced tissue straining on distensible substrates (Morrison et al., 1998a; DeRidder et al., 2001). Given this nearly constant duration, the shear rate in the NSI model increased proportional to increasing peak elongations. Several in vitro TBI models allow independent control of both, strain and strain rate (Ellis et al., 1995; Cargill and Thibault, 1996; Morrison et al., 2000; De-
Ridder et al., 2001; Geddes and Cargill, 2001; Lusardi et al., 2004; LaPlaca et al., 2005). In the present NSI model, the effect of strain rate on cell death can feasibly be explored in future studies by modulating the stiffness of the impact damper, although this would not likely allow for an independent control of both the shear elongation magnitude and rate.

Notwithstanding these limitations, the presented NSI model employed, for the first time, shear strain as the primary mode of mechano-trauma in a controlled and scalable manner. The mechanical insult has been quantified by direct measurement of the inertia-driven transient shear deformation of the organotypic culture during impact. The model uniquely simulates neural shear injury by employing a quantitative and clinically relevant shear insult in vitro, while maintaining a high degree of biological complexity and structural organization in organotypic cultures. As such, the model is well suited for systematic investigation of tissue-level injury thresholds and post-traumatic injury cascades in a controlled environment.

\section{ACKNOWLEDGMENTS}

This work was supported by a grant from the National Institutes of Health (NINDS-R01-NS42946) and a Legacy Research Foundation Grant.

\section{REFERENCES}

ABDEL-HAMID, K.M., and TYMIANSKI, M. (1997). Mechanisms and effects of intracellular calcium buffering on neural survival in organotypic hippocampal cultures exposed to anoxia/aglycemia or to excitotoxins. J. Neurosci. 17, 3538-3553.

ADAMCHIK, Y., FRANTSEVA, M.V., WEISSPAPIR, M., CARLEN, P.L., and PEREZ-VELAZQUEZ, J.L. (2000). Methods to induce primary and secondary traumatic damage in organotypic hippocampal slice cultures. Brain Res. Prot. 5, 153-158.

BAHR, B.A., KESSLER, M., RIVERA, S., et al. (1995). Stable maintenance of glutamate receptors and other synaptic components in long-term hippocampal slices. Hippocampus 5, 425-439.

BUCHS, P.A., STOPPINI, L., and MULLER, D. (1993). Structural modifications associated with synaptic development in area CA1 of rat hippocampal organotypic cultures. Brain Res. Dev. Brain Res. 71, 81-91.

CARGILL, R.S., 2nd, and THIBAULT, L.E. (1996). Acute alterations in $\left[\mathrm{Ca}^{2+}\right] \mathrm{i}$ in NG108-15 cells subjected to high strain rate deformation and chemical hypoxia: an in vitro model for neural trauma. J. Neurotrauma 13, 395-407. 
CATER, H.L., DAVIS, S.M., MORRISON III, B., and SUNDSTROM, L.E. (2004). Pharmacological profile of traumatic brain injury in organotypic hippocampal rat slice cultures following substrate deformation. Presented at the Annu. Meeting Soc. Neurosci. San Diego.

CURTIS, A.S., FORRESTER, J.V., MCINNES, C., and LAWRIE, F. (1983). Adhesion of cells to polystyrene surfaces. J. Cell Biol. 97, 1500-1506.

DENNEY-BROWN, D., and RUSSEL, W.R. (1943). Experimental cerebral concussion. Brain 64, 93-164.

DERIDDER, M.N., GROSVENOR, A.E., MORRISON, B., and MEANEY, D.F. (2001). Mechanical deformation of organotypic cultures induces cell death via both apoptosis and necrosis pathways. Presented at the 2001 Bioengineering Conference, Snowbird, UT.

ELLIS, E.F., McKINNEY, J.S., WILLOUGHBY, K.A., LIANG, S., and POVLISHOCK, J.T. (1995). A new model for rapid stretch-induced injury of cells in culture: characterization of the model using astrocytes. J. Neurotrauma 12, 325-339.

FINSEN, B.R., TONDER, N., AUGOOD, S., and ZIMMER, J. (1992). Somatostatin and neuropeptide $Y$ in organotypic slice cultures of the rat hippocampus: an immunohistochemical and in situ hyridization study. Neuroscience 47, 105-113.

FUNG, Y.C. (1994). A First Course in Continuum Mechanics: For Physical and Biological Scientists and Engineers, 3rd ed. Prentice-Hall: London.

GAEHWILER, H., CAPOGNA, M., DEBANNE, D., McKINNEY, R.A., and THOMPSON, S.M. (1997). Organotypic slice cultures: a technique has come of age. Trends Neurosci. 20, 471-477.

GEDDES, D.M., and CARGILL, R.S., 2nd (2001). An in vitro model of neural trauma: device characterization and calcium response to mechanical stretch. J. Biomech. Eng. 123, 247-255.

GENNARELLI, T.A., THIBAULT, L.E., ADAMS, J.H., GRAHAM, D.I., THOMPSON, C.J., and MARCINCIN, R.P. (1982). Diffuse axonal injury and traumatic coma in the primate. Ann. Neurol. 12, 564-574.

GUTIERREZ, E., HUANG, Y., HAGLID, K., et al. (2001). A new model for diffuse brain injury by rotational acceleration: I. Model, gross appearance, and astrocytosis. J. Neurotrauma 18, 247-257.

HAILER, N.P., JARHULT, J.D., and NITSCH, R. (1996). Resting microglial cells in vitro: analysis of morphology and adhesion molecule expression in organotypic hippocampal slice cultures. Glia 18, 319-331.

KING, A.I., RUAN, J.S., ZHOU, C., HARDY, W.N., and KHALIL, T.B. (1995). Recent advances in biomechanics of brain injury research: a review. J. Neurotrauma 12, 651-658.

KING, A.I., YANG, K.H., ZHANG, L., and HARDY, W. (2003). Is head injury caused by linear or angular acceleration? Presented at the IRCOBI Conf.
KRIMER, L.S., and GOLDMAN-RAKIC, P.S. (1997). An interface holding chamber for anatomical and physiological studies of living brain slices. J. Neurosci. Methods 75, 55-58.

LAPLACA, M.C., LEE, V.M., and THIBAULT, L.E. (1997). An in vitro model of traumatic neuronal injury: loading ratedependent changes in acute cytosolic calcium and lactate dehydrogenase release. J. Neurotrauma 14, 355-368.

LAPLACA, M.C., CULLEN, D.K., McLOUGHLIN, J.J., and CARGILL, R.S., 2nd (2005). High rate shear strain of threedimensional neural cell cultures: a new in vitro traumatic brain injury model. J. Biomech. 38, 1093-1105.

LIGHTHALL, J.W., DIXON, C.E., and ANDERSON, T.E. (1989). Experimental models of brain injury. J. Neurotrauma 6, 83-97.

LUCAS, J.H., and WOLF, A. (1991). In vitro studies of multiple impact injury to mammalian CNS neurons: prevention of perikaryal damage and death by ketamine. Brain Res. 543, 181-193.

LUSARDI, T.A., RANGAN, J., SUN, D., SMITH, D.H., and MEANEY, D.F. (2004). A device to study the initiation and propagation of calcium transients in cultured neurons after mechanical stretch. Ann. Biomed. Eng. 32, 1546-1558.

MEANEY, D.F., SMITH, D.H., SHREIBER, D.I., et al. (1995). Biomechanical analysis of experimental diffuse axonal injury. J. Neurotrauma 12, 689-694.

MEANEY, D.F., ROSS, D.T., WINKELSTEIN, B.A., et al. (1994). Modification of the cortical impact model to produce axonal injury in the rat cerebral cortex. J. Neurotrauma 11, 599-612.

MIELKE, J.G., COMAS, T., WOULFE, J., MONETTE, R., CHAKRAVARTHY, B., and MEALING, G.A. (2005). Cytoskeletal, synaptic, and nuclear protein changes associated with rat interface organotypic hippocampal slice culture development. Brain Res. Dev. Brain Res. 160, 275-286.

MORRISON, B., MEANEY, D.F., and McINTOSH, T.K. (1998a). Mechanical characterization of an in vitro device designed to quantitatively injure living brain tissue. Ann. Biomed. Eng. 26, 381-390.

MORRISON, B., 3rd, SAATMAN, K.E., MEANEY, D.F., and McINTOSH, T.K. (1998b). In vitro central nervous system models of mechanically induced trauma: a review. J. Neurotrauma 15, 911-928.

MORRISON, B.R., MEANEY, D.F., MARGULIES, S.S., and McINTOSH, T.K. (2000). Dynamic mechanical stretch of organotypic brain slice cultures induces differential genomic expression: relationship to mechanical parameters. J. Biomech. Eng. 122, 224-235.

MUHKIN, A.G., IVANOV, S.A., KNOBLAUCH, S.M., and FADEN, A.I. (1997). New in vitro model of traumatic neuronal injury: evaluation of secondary injury and glutamate receptor-mediated neurotoxicity. J. Neurotrauma 14, 651-663. 


\section{MODELING NEURAL INJURY IN ORGANOTYPIC CULTURES}

OMMAYA, A.K., and GENNARELLI, T.A. (1974). Cerebral concussion and traumatic unconsciousness. Correlation of experimental and clinical observations of blunt head injuries. Brain 97, 633-654.

SMITH, D.H., WOLF, J.A., LUSARDI, T.A., LEE, V.M.Y., and MEANEY, D. (1999). High tolerance and delayed elastic response of cultured axons to dynamic stretch injury. J. Neurosci. 19, 4263-4269.

STEELE, J.G., McFARLAND, C., DALTON, B.A., JOHNSON, G., EVANS, M.D., HOWLETT, C.R., and UNDERWOOD, P.A. (1993). Attachment of human bone cells to tissue culture polystyrene and to unmodified polystyrene: the effect of surface chemistry upon initial cell attachment. J. Biomater. Sci. Polym. Ed. 5, 245-257.

STOPPINI, L., BUCHS, P.A., and MULLER, D. (1991). A simple method for organotypic cultures of nervous tissue. J. Neurosci. Methods 37, 173-182.
STOPPINI, L., DUPORT, S., and CORREGES, P. (1997). A new extracellular multirecording system for electrophysiological studies: application to hippocampal organotypic cultures. J. Neurosci. Methods 72, 23-33.

ZHANG, L., BAE, J., HARDY, N., et al. (2002). Computational study of the contribution of the vasculature on the dynamic response of the brain. Stapp Car Crash J. 46, $145-164$.

Address reprint requests to: Michael Bottlang, Ph.D. Biomechanics Laboratory Legacy Research Center $1225 \mathrm{NE} 2^{\text {nd }}$ Avenue Portland, OR 97232

E-mail: mbottlang@biomechresearch.org 